\title{
How Digital and Physical Care Team Interaction Affect Processing Times: A Case Study of Hospitalists
}

\author{
Gurvich $\mathrm{I}^{1}$, Wang $\mathrm{L}^{2}$, O'Leary $\mathrm{KJ}^{3}$, Soulakis $\mathrm{ND}^{3}$, and Van Mieghem $\mathrm{JA}^{\star 2}$ \\ ${ }^{1}$ School of Operations Research and Information Engineering, Cornell University, New York, USA \\ ${ }^{2}$ Kellogg School of Management, Northwestern University, Evanston, USA \\ ${ }^{3}$ Feinberg School of Medicine, Northwestern University, Evanston, USA
}

${ }^{*}$ Corresponding author: Van Mieghem JA, Kellogg School of Management, Northwestern University, Evanston, IL 60208, USA, Tel: +1(847)491 5481, E-mail: vanmieghem@northwestern.edu

Citation: Gurvich I, Wang L, O’Leary KJ, Soulakis ND, Van Mieghem JA (2017) How Digital and Physical Care Team Interaction Affect Processing Times: A Case Study of Hospitalists. J Case Rep Stud 5(6): 606

Received Date: November 09, 2017 Accepted Date: December 27, 2017 Published Date: December 29, 2017

\begin{abstract}
Importance: Hospitalist physicians face increasing pressure to maximize productivity while maintaining high quality of care. Their success, however, depends on the effective exchange of information among a patient's care team. The latter comprises the digital team (caregivers who document in - not just access-the patient's electronic health record) and a physical team (caregivers who directly communicate with the hospitalist).
\end{abstract}

Objective: To determine the association between hospitalist total daily processing time per patient and the size and evolution over the patient's length of stay (LOS) of the digital and physical teams as well as patient-level characteristics.

Design: We measured hospitalist daily processing times and captured the physical team through a time-and-motion study of hospitalists. The digital team interactions were extracted from patient Electronic Health Records.

Setting: Northwestern Memorial Hospital, a large academic urban hospital in Chicago. Participants Our case study selected four hospitalists at random who cared for 107 inpatient stays over 17 days in June-July 2014 and collaborated with 2046 caregivers: 301 were observed physical collaborators and 1745 were digital-only collaborators.

Exposures: Hospitalist activities and patient encounters from observations and electronic health records.

Main Outcomes and Measures: Processing time is the total time spent by the hospitalist on a patient's care per day. Key covariates are patient-level characteristics, interruptions by physical team members, and size and evolution of both the digital and physical teams. Results Teamwork interaction and patient-level characteristics explain $72 \%$ (18.9min) and 28\% (7.2min), respectively, of the hospitalist's average patient processing time of $26.7 \mathrm{~min}$ per day. Teamwork is further decomposed in two ways: (i) $18.9=15.4+3.5$ where $15.4 \mathrm{~min}$ and $3.5 \mathrm{~min}$ captures the teamwork effect at a macro level (driven by team size and stability variables) and micro level effect (hospitalist workflow interruptions driven by task switches), respectively; (ii) $18.9=8.8+10.1$ where $8.8 \mathrm{~min}$ and $10.1 \mathrm{~min}$ capture digital and physical team variables, respectively. As a benchmark, eliminating interruptions reduces processing times by $3.5 \mathrm{~min}$ (13\%) while minimizing team size and maximizing team stability reduces processing times by $7.8 \mathrm{~min}(29 \%)$.

Conclusion and Relevance: Hospitalist processing times are impacted as much by the digital as the physical care team characteristics. Although our findings should be validated in different clinical settings, they suggest the need to combine digital data with observational data to evaluate hospitalist processing times and to mitigate the negative effects of interruptions and care team turnover.

Keywords: Daily Processing Times; Time-And-Motion Study; Electronic Health Records

\section{Background}

Interaction and teamwork are fundamental characteristics of healthcare. Both bring benefits but also introduce costs: large volumes of information must be processed and information exchanges often interrupt individual workflow. We adopt a process perspective of work consisting of sequenced activities and use processing time, the total time spent by a specific caregiver on a patient's care per day, as a concrete and precise measure of these costs. We present a novel approach to quantify the impact of both conventional physical and contemporary digital interaction on processing times.

Healthcare often is a complex, resource and information intensive process: by the end of a hospitalization, a patient's care team comprises dozens and sometimes hundreds of individuals [1-3]. While caregivers input is essential to patient care, the size and volatility of these teams put a burden on the hospitalists: the physicians that act as information hubs and collect information to 
support diagnosis and coordinate patient care plans [4,5]. Effective exchange of information among team members is essential to high quality of care. Information is exchanged through interpersonal communication (e.g., a text message, a phone call or a faceto-face meeting) or digital documentation (e.g., a physician entering a prescription in a patient's file or a pharmacist subsequently fulfilling it).

Each working day, the hospitalist diagnoses patients, determines treatment plans (laboratory tests, surgeries, medications, etc.), communicates with other caregivers to guide diagnosis and treatment and summarizes all relevant information in the patient's medical chart [6]. Whereas the number of patients determines the hospitalist work efficiency and care quality [7], the team that the hospitalist interacts with also influences processing time. The patient's digital team consists of caregivers that document information in the patient's Electronic Health Record (EHR) and contribute to the patient's care knowledge network [8,9]. (Note that digital team members edit the EHR; we exclude people who only read or access the EHR). The hospitalist processes the digital information and augments it after interacting with her physical team. The latter consists of caregivers who we observed interact with the hospitalist, who serves as the focal physical team member, using interpersonal communications regarding the patient. The total team is a network with two layers (Figure 1). The digital layer captures the patient's documenta- tion team and the physical layer captures the hospitalist's interpersonal communication team. The figure presents two snapshots of the total team of a given patient and a given hospitalist.

Admission day (2014-07-07)

Discharge day (2014-07-12)
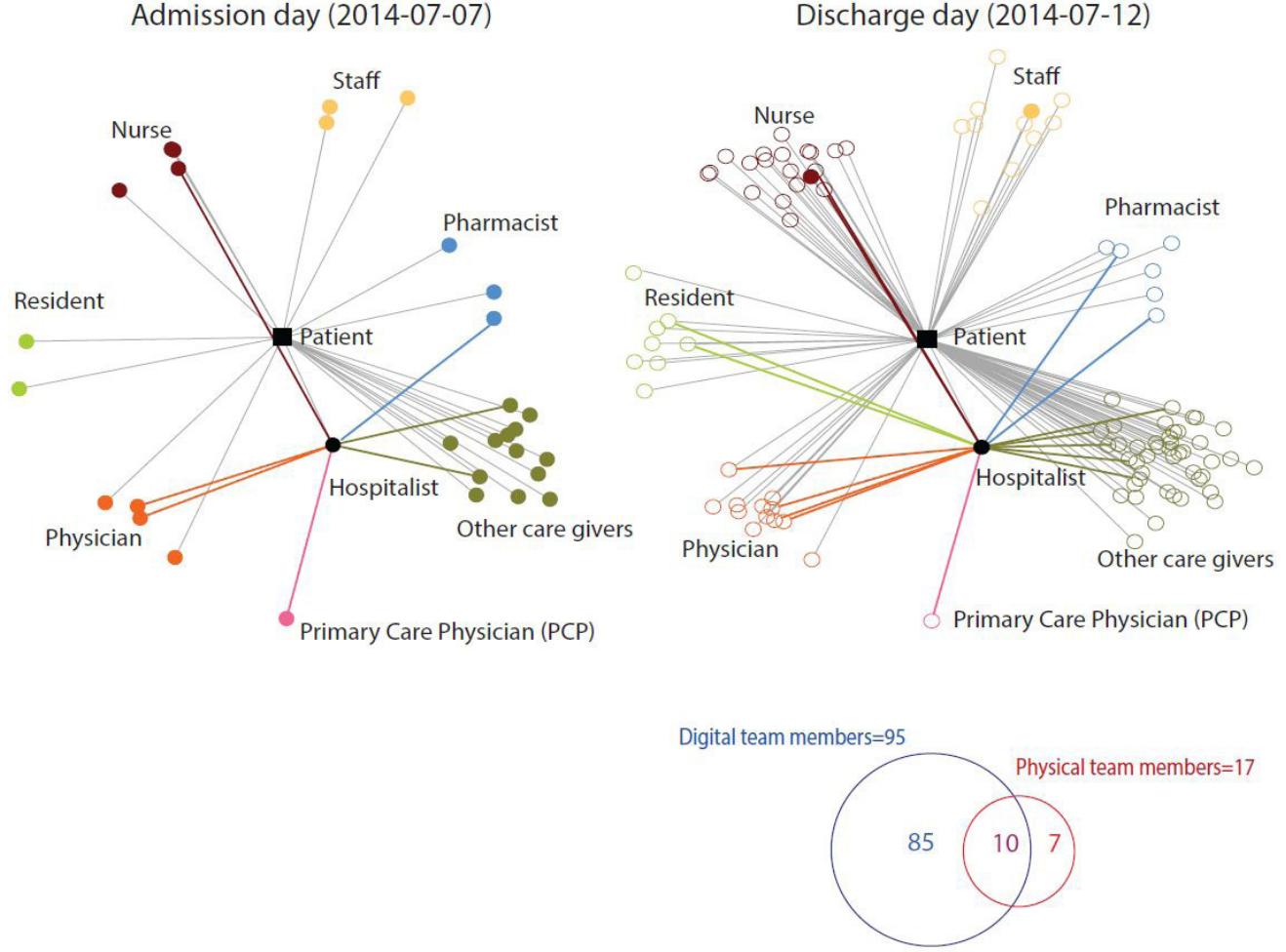

Figure 1: Team interaction evolution: a patient's digital team and the hospitalist's physical team. Each filled circle represents a care provider active on that day; unfilled circles were active on prior days. Care providers with different titles are connected to the patient (rectangular node) with grey edges (digital layer) if they enter digital information in the patient EHR. They are connected to the hospitalist with colored edges (physical layer) if we observed them communicating with the hospitalist regarding the patient. The entire care team of this patient with the hospitalist as the focal member after 6 observation days included 102 caregivers and consisted of 95 digital and 17 physical team members - 10 providers both input data to the patient EHR and were observed communicating with the hospitalist

Our new approach quantifies and benchmarks the day-by-day and patient-by-patient effects of the physical and digital layers on the hospitalist's processing time. Our model incorporates both micro and macro levels of teamwork. At the micro level we capture interaction-driven interruptions to the hospitalist's workflow. At the macro level we capture stability which is often defined using the number or fraction of new members $[10,11]$. We decompose the digital and the physical teams on each day into three membership categories (Figure S1) [12]: cumulative members have membership prior to the beginning of that day (i.e., they have performed at least one patient activity prior to that day); daily members have active membership on that day; and new members are daily members that enter the team that day. The model captures team member familiarity by counting past interactions of the hospitalist with cumulative team members. It captures task familiarity through membership of the patient's team in previous days.

Using a case study, we demonstrate how mode of interaction (digital documentation or interpersonal communication) and team scale and stability (through team size, task and member familiarity) affect hospitalist processing times. In the empirical literature, team size as well as member- and task-familiarity are often measured at the conclusion of a team's project; the theoretical literature models and analyzes aspects of team evolution [13-20]. Our novelty lies in studying how hospitalist processing times are affected not only by her physical, but also her digital, team. 


\section{Data}

Hospitalist daily processing time is the total daily time s/he spends on all activities related to one patient. It is determined by patient and hospitalist characteristics as well as by the exchange of information with other members of the patient's care team.

We measured processing times through careful observation, which yields our first data set: a time-motion study that we conducted at Northwestern Memorial Hospital (NMH) over 17 days in June-July, 2014. We observed four hospitalists and recorded all their individual activities and interpersonal communications with team members. Hospitalists work in rotations with on and off periods of 7 days. For each day, we shadowed one hospitalist during an entire shift. The data captures patient identifiers, activities, and names and titles of care providers with whom the hospitalist interacted—this identifies the physical team (Table S1) [21].

Our second data set is from the hospital's EHR. Each patient's EHR includes forms, notes and other transactions (e.g. surgeries, food intake, medication, lab) ordered and performed throughout the patient's stay at the hospital. Each recorded transaction has a time stamp and the ID of the provider that entered the transaction. Only providers that edit the EHR are part of the patient's digital team.

Both data sources complement each other to provide a comprehensive picture of the total team and of every hospitalist activity [22]. The latter are necessary to measure hospitalist processing time; this requires observational data because many hospitalist activities, including some interpersonal communications, leave no footprint in the EHR.

We merged the two data sets by patient identifier and day of activity so that our unit of analysis is (patient, day). Our study covers 17 observation days and 109 patient encounters, totaling 229 observed patient-day assignments (each to a unique hospitalist). We observed 301 caregivers who physically communicated with the four hospitalists about the care of these 109 patients (Figure 2); an additional 1745 caregivers made entries in those patients' EHR but never physically communicated with the four hospitalists (Figure 3). Table 1 reports summary statistics for patient, hospitalist workflow and team characteristics. The mean observed processing time is $26.1 \mathrm{~min}$ with a standard deviation of $15.0 \mathrm{~min}$. The mean communication time is $6.3 \mathrm{~min}$ with a standard deviation of $7.7 \mathrm{~min}$. In terms of interruptions, the hospitalist preempts the documentation of a patient's case 3.5 times on average, 1.9 times to switch to reaching out to other individuals for communication, and 1.6 times to switch to responding to other individuals communication requests.

\section{Observed Communication Network = Physical Team}

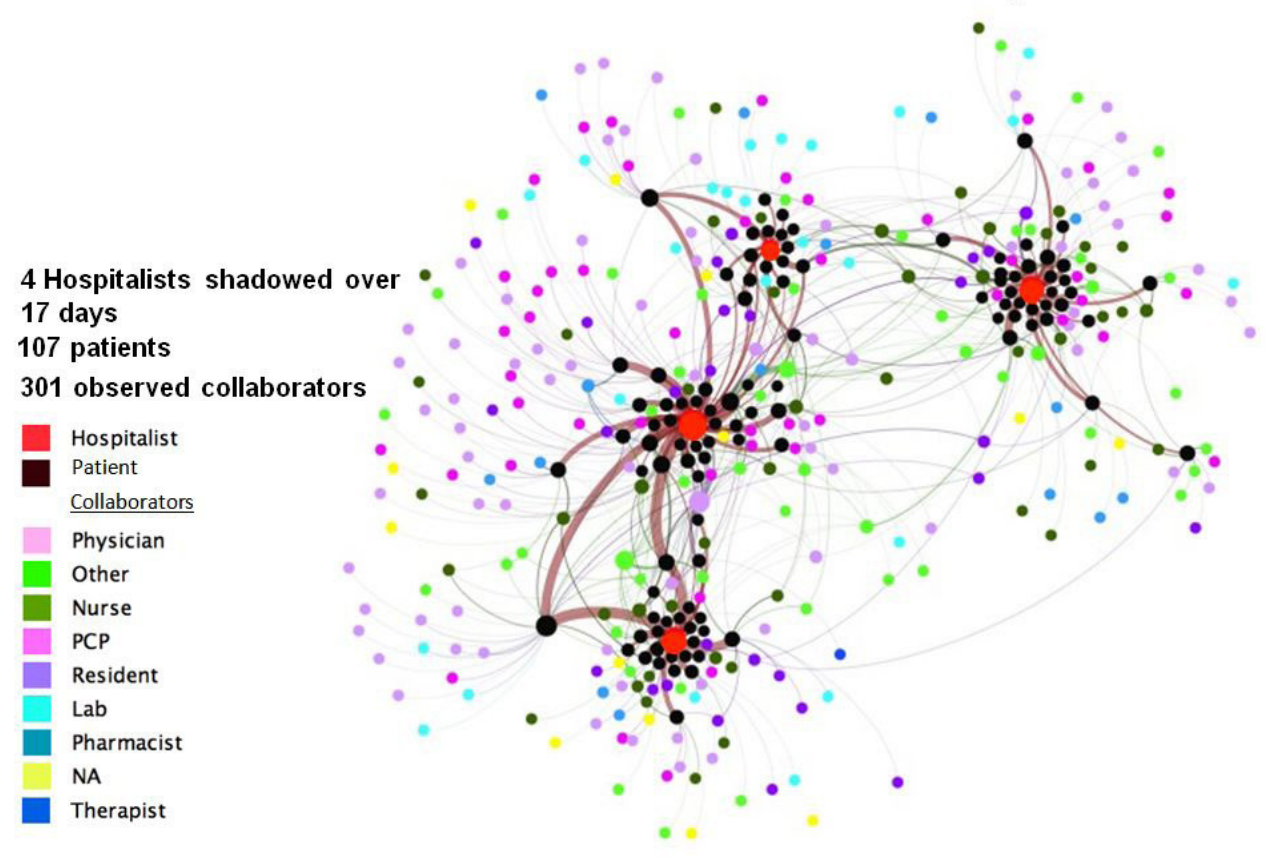

Figure 2: The physical team comprises 301 caregivers with whom we observed the hospitalists communicate during our case study. The width of an arc represents communication frequency

Physical teams are generally smaller than digital teams: the cumulative physical and digital team sizes on a patient's discharge day are 7 and 82, respectively. The largest cumulative digital team has 331 members, 14 times that of the largest physical team size that had 26 members. On average (over patient and days), the digital daily team has 21 members while the physical daily team has 4 . On average, the digital team is more unstable with $70 \%$ of its members being new relative to $50 \%$ for the physical team.

Observed Communication Network = Physical Team 


\begin{tabular}{|c|c|c|c|}
\hline & Source $^{1}$ & Median (Min,Max) & Mean (SD) \\
\hline \multicolumn{4}{|l|}{ Dependent variable } \\
\hline Processing time (in minutes) & OBS & $24(5,101)$ & $26.1(15.0)$ \\
\hline Communication time (in minutes) & OBS & $4(0,49)$ & $6.3(7.7)$ \\
\hline \multicolumn{4}{|l|}{ Coded patient characteristics $^{2}$} \\
\hline Acuity level & EHR & $3(1,5)$ & $3(1.0) \mathrm{ICU}$ \\
\hline indicator & EHR & $0(0,1)$ & $0.2(0.4)$ \\
\hline Length of Stay (hours) & EHR & $120(15,768)$ & $204(213.8)$ \\
\hline PCP-NMH-employment? & EHR & $1(0,1)$ & $0.6(0.5)$ \\
\hline Discharge today? & EHR & $0(0,1)$ & $0.3(0.4)$ \\
\hline Patient-Hospitalist familiarity & EHR & $2(1,5)$ & $1.9(1.0)$ \\
\hline $\begin{array}{l}\text { Time when the focal patient EHR starts being } \\
\text { documented }\end{array}$ & OBS & $11(7,18)$ & $11.7(2.3)$ \\
\hline $\begin{array}{l}\text { Number of other patient EHRs in pipeline } \\
\text { While documenting the focal EHR: }\end{array}$ & OBS & $3(0,11)$ & $3.4(2.4)$ \\
\hline \multicolumn{4}{|l|}{ Hospitalist workflow characteristics } \\
\hline \# of task switches to reach out & OBS & $1(0,10)$ & $1.9(2.2)$ \\
\hline \# of task switches to respond & OBS & $1(0,9)$ & $1.6(2.0)$ \\
\hline \multicolumn{4}{|l|}{ Team size at discharge $^{3}$} \\
\hline Physical team ${ }^{4}$ size & OBS & $5(1,26)$ & $7(4)$ \\
\hline Digital team $^{5}$ size & EHR & $60(23,331)$ & $82(61)$ \\
\hline \multicolumn{4}{|l|}{$\begin{array}{l}\text { Team variables-Team membership catego- } \\
\text { ries }^{6}\end{array}$} \\
\hline Physical cumulative team size & OBS & $2(0,21)$ & $3(4)$ \\
\hline Digital cumulative team size & EHR & $44(0,294)$ & $70(71)$ \\
\hline Physical daily team size & OBS & $3(1,12)$ & $4(2.1)$ \\
\hline Digital daily team size & EHR & $20(6,53)$ & $21(8.4)$ \\
\hline Physical new team fraction & OBS & $0.8(0,1)$ & $0.7(0.3)$ \\
\hline Digital new team fraction & EHR & $0.5(0,1)$ & $0.5(0.3)$ \\
\hline \multicolumn{4}{|l|}{ Team variables-Collaboration experience } \\
\hline Collaboration experience & EHR & $5(0,18)$ & $5(4)$ \\
\hline
\end{tabular}

${ }^{1}$ Two sources of data: observed from time-motion study-OBS, extracted from the Electronic Healthcare Records-EHR.

${ }^{2}$ Acuity level, assessed by the admitting physician, ranges from 1 to 5 , with 5 being the most acute; ICU indicator equals to 1 if the patient is of ICU status and 0 if not; PCP employed by NMH and Discharge today equal to 1 if the answer to either question is yes and 0 otherwise. Time of starting documenting the focal patient EHR is measured in hours of the day; Multitasking level is measured by number of patient EHR documentations that have been started but not yet finished upon starting the focal patient EHR.

${ }^{3}$ Team size at discharge counts the number of care providers accumulated by the time the patient is discharged. ${ }^{4}$ Physical team consists of care providers that have interpersonal communications with the hospitalist regarding the patient during our observation.

${ }^{5}$ Digital team consists of care providers that have inputted digital documentations in the patient's EHR.

${ }^{6}$ Each team membership category is measured regarding the unit of analysis-(patient, day).

Table 1: Descriptive statistics of the dependent variables, patient and hospitalist characteristics

\section{Team Evolution Model}

We adopt a linear model of how hospitalist processing time is impacted by patient characteristics, hospitalist workflow, and team variables. These covariates may be correlated; e.g., the larger the physical team, the more interruptions one expects to the hospitalist workflow. To re- solve multicollinearity, we perform factor analysis [20] to group highly correlated variables into a smaller set of independent factors. We shall see that this statistical procedure groups original variables into factors that naturally correspond to team scale, team stability and workflow interruptions.

We denote the covariates [23] as (P, W, T), where $\mathbf{P}$ include patient characteristics-Acuity level [24], Intensive Care Unit (ICU) indicator, Length of Stay, the indicator of whether the patient's primary care physician (PCP) is employed by $\mathrm{NMH}$, the indicator of the patient being discharged on the focal day, Patient-Hospitalist familiarity, Time of day and number of other patient EHRs in pipeline when the patient EHR starts being documented; W includes hospitalist workflow characteristics-Number of task switches to reach out and number of task switches to respond; $\mathbf{T}$ includes team variables-digital and physical cumulative, daily team sizes, new team member fractions, and the hospitalist's collaboration experience with the team. 


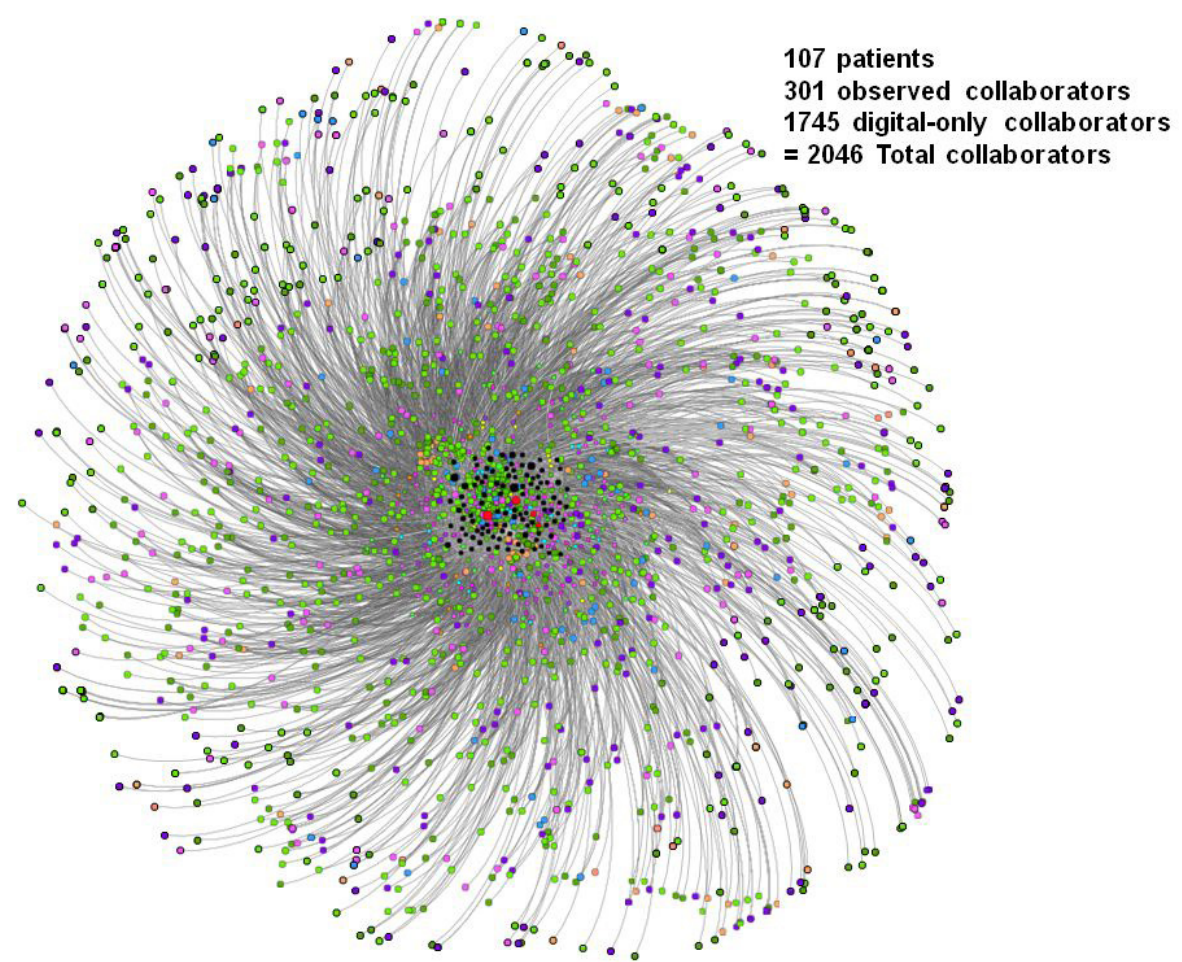

Figure 3: This plot superimposes the 1745 digital-only collaborators on the 301 physical collaborators of the hospitalists (displayed in Figure 2) during our case study

The analysis groups patient characteristics into factors $F$ such that $\mathbf{P}=\mathbf{L F}$, subject to $C O V(\mathbf{F})=\mathbf{I}$, where $\mathbf{I}$ and $\mathbf{L}$ are the identity and loading factor matrix, respectively. Hospitalist and team characteristics are grouped into factors $\mathbf{G}$ such that $[\mathbf{W}, \mathbf{T}]=\mathbf{M G}$, subject to $\operatorname{COV}(\mathbf{G})=\mathbf{I}$, where $\mathbf{M}$ is the loading factor matrix.

Table 2 reports the results of factor analyses allowing for 3 factors for team and workflow variables and 2 factors for patient variables. (For robustness we varied the number of factors and obtained similar results; c.f. Table S2 [25].) Given this number of factors, the factoring method [26] returns the "best" grouping of variables:

\begin{tabular}{|l|c|}
\hline \multicolumn{1}{|c|}{ Variable } & Factor loading \\
\hline $\begin{array}{l}\text { (a) Team and Hospitalist workflow } \\
\text { characteristics }\end{array}$ & 3 Factors \\
\hline Digital cumulative team size & Factor 1 \\
\hline Digital new-member fraction & -0.8 \\
\hline Physical cumulative team size & 0.7 \\
\hline & Factor 2 \\
\hline \# of switches to reach out & 0.8 \\
\hline \# of switches to respond & 0.6 \\
\hline Physical daily team size & 0.7 \\
\hline & Factor 3 \\
\hline Digital daily team size & 0.7 \\
\hline Physical new-member fraction & 0.4 \\
\hline Collaboration experience & -0.4 \\
\hline Factor analysis statistics & \\
\hline p-value & \\
\hline (b) Patient characteristics & $\mathbf{2 ~ F a c t o r s ~}$ \\
\hline & Factor 1 \\
\hline Discharge today? & -0.4 \\
\hline Length of Stay & 0.9 \\
\hline ICU indicator & 0.5 \\
\hline
\end{tabular}




\begin{tabular}{|l|c|}
\hline \multicolumn{1}{|c|}{ Variable } & Factor loading \\
\hline & Factor 2 \\
\hline \# of days seen by the hospitalist & 1.0 \\
\hline $\begin{array}{l}\text { Time of day the focal patient EHR } \\
\text { starts being documented }\end{array}$ & \\
\hline Acuity level & \\
\hline PCP employed by NMH? & \\
\hline Factor analysis statistics & 0.1 \\
\hline p-value & \\
\hline
\end{tabular}

${ }^{1}$ The null hypothesis is that the number of factors constrained is sufficient.

Table 2: Orthogonal rotated factor pattern (|Loadings $\mid>=0.4$ )

Factor $1\left(G_{1}\right)$ captures that the cumulative physical and digital team sizes are positively associated with each other. We will naturally refer to this factor as the team size factor. G1 is also captures that cumulative digital team size is negatively associated with the fraction of new digital team members. Large digital teams occur near the end of a patient's length of stay at which point few members join the team.

Factor $2\left(G_{2}\right)$ captures the positive association among the number of switches to reach out, the number of switches to respond, and physical daily team size. We will refer to this factor as the workflow factor.

Factor $3\left(G_{3}\right)$ captures the negative association between physical new-member fraction and collaboration experience. We will refer to this factor as the team unfamiliarity or instability factor. $G 3$ also captures that digital daily team size is positively associated with physical new- member fraction. The hospitalist may reach out to digital daily team members to discuss their digital entries; if they were not yet members of the cumulative physical team, they become new members of the physical team.

The "best" grouping of the patient variables is shown in the bottom part of Table 2 . The $p$-value of 0.1 of the factor analysis suggests eliminating Acuity level, PCP-NMH-employment indicator, Time of day the patient EHR starts being documented from the model and grouping the remaining variables into 2 factors:

Factor $1\left(F_{1}\right)$ shows that Intensive Care Unit patients have a longer length of stay (LOS) and are less likely to be discharged on a particular day. We will refer to this factor as the LOS factor.

Factor $2\left(F_{2}\right)$ equals the number of days the patient has been seen by the hospitalist, and will be called the hospitalist-patient familiarity factor.

Having formally derived the scale, stability and workflow factors $G$ and the patient LOS and familiarity factors $F$, the team evolution model is a weighted ${ }^{1}$ Generalized Linear Model (GLM) [27] of the log of the hospitalist processing and communication time. (The logarithm is used because both times are indeed Weibull distributed, as often assumed in duration models.) The regression equations for patient $i$ on day $t$ are:

$$
\begin{aligned}
& \log \left(\text { processing time }_{\mathrm{i}, \mathrm{t}}\right)=\alpha_{\mathrm{i}, \mathrm{t}}{ }^{1}+\beta^{1} \mathrm{~F}_{\mathrm{i}, \mathrm{t}}+\mathrm{Y}^{1} \mathrm{G}_{\mathrm{i}, \mathrm{t}}+\mathrm{U}_{\mathrm{i}, \mathrm{t}} \\
& \log \left(\text { communication time }_{\mathrm{i}, \mathrm{t}}+1\right)=\mathrm{a}_{\mathrm{i}, \mathrm{t}}{ }^{2}+\beta^{2} \mathrm{~F}_{\mathrm{i}, \mathrm{t}}+\mathrm{Y}^{2} \mathrm{G}_{\mathrm{i}, \mathrm{t}}+\mathrm{V}_{\mathrm{i}, \mathrm{t}}
\end{aligned}
$$

\section{Results}

\section{The Effects of Teamwork on Processing Times}

Estimation of the coefficients $\alpha, \beta$ and $\gamma$ in the linear team evolution model (Equations (1) and (2)) gives first insights of the impact of the patient factors $\mathrm{F}$ and team and workflow factors $\mathrm{G}$ on the hospitalist processing times. Table 3 reports the regression results (Table S3 [28]). The left column shows that the hospitalist processing time increases with an increase in the team size factor $G_{1}$, workflow factor $G_{2}$, and team instability factor $G_{3}$ and decreases when hospitalist-patient familiarity $F_{2}$ increases. Other patient characteristics $\left(F_{1}\right)$ have no statisti- cally significant impact on hospitalist processing time. The right column shows that the hospitalist communication time increases only with an increase in workflow factor $G_{2}$, while team size, stability and other patient factors have no statistically significant impact.

Recall that workflow factor $G_{2}$ includes physical daily team size and task switches and reflects the communication burden which indeed increases communication and processing times. The impact of task switches agrees with the finding in [29] that hospitalist documenting time (a part of the processing time) increases with the switching frequency due to a setup time for memory retrieval.

\footnotetext{
${ }^{1}$ We include a weight in the regression for each data point to deal with potential confounding effects-patient and hospitalist characteristics may impact both the hospitalist processing time and the task switching frequency needed for communication [29]. The weight is obtained with the Propensity Score Weighting Method [30]: the data is grouped into three subsets: the control group with no task switches, the treatment group A with 1 to 3 task switches, and the treatment group B with 4 or more switches. The propensity score for each data point is equivalent to the probability of that data point falling into the control group if all patient characteristics input variables were randomly distributed among all data.
} 
An increase in team size factor $G_{1}$ reflects the increased team information load that the hospitalist must acquire and process, which increases processing times, in agree ment with the positive coefficient 0.24 . Finally, the positive coefficient 0.19 of $\mathrm{G}_{3}$ agrees with that factors' interpretation: higher unfamiliarity among the team requires a larger processing time by the hospitalist to integrate information across the team. Team familiarity, however, has no significant impact on communication time.

\begin{tabular}{|c|c|c|}
\hline Variable & \multicolumn{2}{|c|}{ Estimated coefficient $\left(\mathrm{SD}^{1}\right)$} \\
\hline Dependent variable & $\begin{array}{c}\text { Equation }(\mathbf{1}) \\
\log (\text { Processing time })\end{array}$ & $\begin{array}{c}\text { Equation }(2) \\
\log (\text { Communication time }+\mathbf{1})\end{array}$ \\
\hline Intercept & $3.14(0.04)^{\star * *}$ & $1.69(0.08)^{\star * *}$ \\
\hline \multicolumn{3}{|l|}{ Patient characteristics $^{2}$} \\
\hline $\mathrm{F}_{1}$ & $-0.12(0.10)$ & $0.14(0.23)$ \\
\hline $\mathrm{F}_{2}$ & $-0.14(0.06)^{*}$ & $-0.02(0.13)$ \\
\hline \multicolumn{3}{|l|}{$\begin{array}{c}\text { Team/Hospitalist } \\
\text { workflow }^{3}\end{array}$} \\
\hline $\mathrm{G}_{1}$ & $0.24(0.10)^{*}$ & $-0.01(0.25)$ \\
\hline $\mathrm{G}_{2}$ & $0.50(0.06)^{* * *}$ & $0.95(0.10)^{* * *}$ \\
\hline $\mathrm{G}_{3}$ & $0.19(0.05)^{\star * *}$ & $0.10(0.11)$ \\
\hline \multicolumn{3}{|c|}{$\begin{array}{l}{ }^{1} \text { We calculate the standard deviations of predictions with bootstrap regressions. } \\
{ }^{2} F_{1}: \text { Discharge today?, Length of stay, ICU indicator; } F_{2}: \text { \# of days the patient has been seen } \\
\text { by the hospitalist. } \\
{ }^{3} G_{1}: \text { Physical/Digital cumulative team size, Digital new-member fraction; } G_{2}: \text { \# of switches to } \\
\text { reach out/respond, Physical daily team size; } G_{3}: \text { Digital daily team size, Physical new-member } \\
\text { fraction, Collaboration experience. } \\
\text { Table 3: Regression results }\end{array}$} \\
\hline
\end{tabular}

\section{Decomposition: Processing times explained by teamwork}

To quantify how hospitalist processing time is impacted by teamwork variables, we use our model to first decompose the observed average daily processing time into two components: time determined by patient characteristics and by teamwork. The parts of the

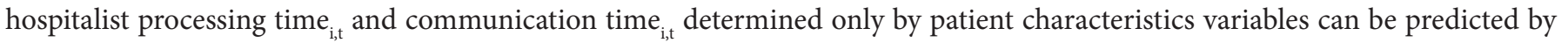
model Equation (1)-(2) when setting all team variables (i.e., the entire factor vector $\mathrm{G}_{\mathrm{i}, \mathrm{t}}$ ) equal to zero. Taking averages results in $7.2 \mathrm{~min}$ (standard deviation ${ }^{2} \mathrm{SD}=1.0 \mathrm{~min}$ ) and $1.3 \mathrm{~min}(\mathrm{SD}=0.5 \mathrm{~min}$ ), respectively (Table S4 [31]).

Our model thus decomposes the observed average processing and communication times as $26.1=7.2+18.9$ and $6.3=1.3+5.0$, respectively. In other words, teamwork interaction explains $18.9 \mathrm{~min}(72 \%)$ and $5.0 \mathrm{~min}(81 \%)$ of the hospitalist average processing and communication time. The Supplemental Materials provide two further decompositions of the teamwork: (i) $18.9=15.4+3.5$ where $15.4 \mathrm{~min}$ captures the teamwork effect at a macro level (driven by team size and stability variables) while $3.5 \mathrm{~min}$ captures the micro level effect of hospitalist workflow interruptions (driven by task switches) ${ }^{3}$; (ii) $18.9=8.8+10.1$ where $8.8 \mathrm{~min}$ and $10.1 \mathrm{~min}$ capture digital and physical team variables, respectively (Table S4 [32]). We could similarly decompose the predicted (rather than observed) dependent variables which leads to the numbers reported in the abstract.

Given this decomposition, one may be tempted to argue that hospitalist processing time could be reduced by $18.9 \mathrm{~min}$. This, however, would require total re-integration of work: collapsing the team into an omniscient specialist who makes all diagnoses without consulting other care providers. The next analysis takes the actual division of labor as given and constructs best and worst case benchmarks.

\section{Benchmarking: Processing time sensitivity to team evolution}

To evaluate the performance of a given team, we use our model to provide three benchmarks by simulating three extreme team evolution scenarios:

i) The benchmark of best possible performance assumes a team evolution with minimal scale and maximal stability: for each patient, the daily team size remains the actual team size that showed up on admission day. This captures the extreme minimal daily team. Consequently on each day, both the daily and cumulative team sizes equal the team size on the admission day, while the new-member fraction $=0$;

ii) One benchmark of worst performance assumes a team evolution with minimal stability: each patient receives an entirely new team every day. Consequently, we keep the actual daily team size but assume all team members are new members: the newmember fraction always $=1$;

iii) Another benchmark of worst performance considers a team evolution with maximal scale and maximal stability: on each day, a patient's team equals the actual team accumulated at discharge. This captures the extreme maximal daily team. On each day, both the daily and cumulative team sizes equal the cumulative team size at discharge, while the new-member fraction $=0$.

${ }^{2}$ We run 1000 bootstrap regressions-resample with replacement within each regression for each column, and obtain corresponding standard deviation for each predicted value.

${ }^{3} 3.5 \mathrm{~min}$ falls in the $95 \%$ confidence interval of mental setup time due to task switching estimated in [29]. 
The left panel of Figure 4 shows the cumulative team size of the three simulated scenarios against the actual team evolution. The horizontal axis marks the admission (day 0 ) and the observed day index ${ }^{4}$. The team evolution with maximal stability yields the smallest team at discharge (17 people). While this benchmark represents an ideal, notice that the average daily team size equals $4+21=25$ according to Table 1 . Therefore, if there were no turnover in the actual team, its cumulative team size would remain constant at 25 , not that far from the benchmark. The team evolution with minimal stability replaces the daily team with new members each day; therefore, it results in the maximal possible cumulative team size at discharge (93 people).
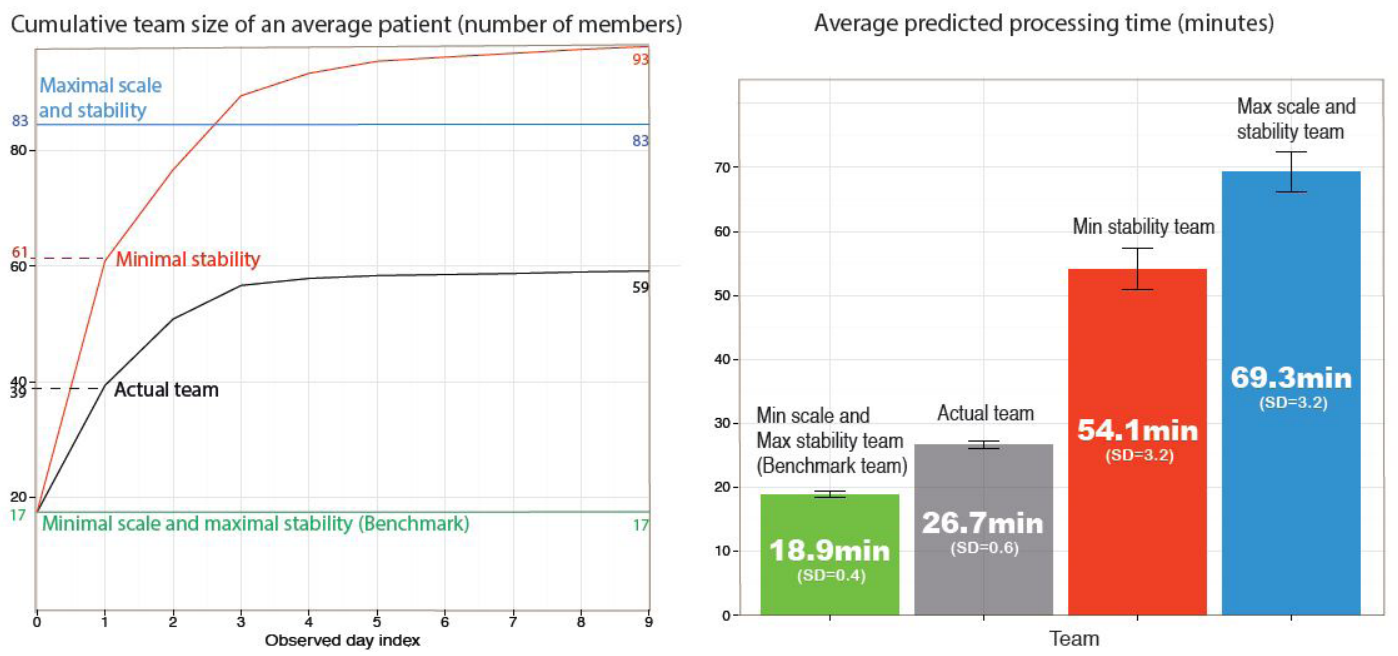

Figure 4: Benchmarking actual performance against 3 simulated team evolution extremes. The left panel shows the average cumulative team size evolution of the actual team against three simulated team evolutions. An average patient's team starts to accumulate on admission (Day 0); Day 1 to Day 9 represents the $1^{\text {st }}$ to $\mathrm{g}^{\text {th }}$ observed day during a patient's length of stay. With an entirely new team each day (minimal stability), an average patient would have 93 team members accumulated on the last observed day, while the actual accumulated team size was 59. The best possible performance would keep the daily team equal to the team at admission (minimal scale and maximal stability)

\section{Right panel}

Our model predicts the average processing time of a patient on a day would be $69.3 \mathrm{~min}$ if the daily team equaled the actual team at discharge (maximal scale) and $54.1 \mathrm{~min}$ for the minimal stability team. The average processing time of the actual team is $26.7 \mathrm{~min}$ versus $18.9 \mathrm{~min}$ under the best possible benchmark team evolution (minimal scale and maximal stability).

The right panel shows our model predictions of the average processing time under the three simulated team evolutions against the actual team evolution [33]. First, notice that the actual performance is fairly good: $26.7 \mathrm{~min}$ is only $41 \%$ above the best benchmark ${ }^{5}$ second; team scale has a larger effect on processing times than team instability in our data.

\section{Discussion and Conclusion}

The goal of this paper is to showcase how to quantify the impact of multiple patient and team variables on an individual hospitalist's processing times. Populated with empirical data of the specific physician's workflow, the model allows objective, data-driven performance evaluation by benchmarking observed processing times against other team size and stability scenarios. We applied this methodology to hospitalists at Northwestern Memorial Hospital. To better understand the demonstrated large effects of team scale and team stability on processing times, we revisit our model to extract further insight.

\section{Team Scale}

The average hospitalist's cumulative physical team is very small (3) relative to the patient's digital team (70). Thus, when considering the factor $G_{1}$ (team scale), it is mostly variations in the cumulative digital team (and not the physical) that underlie the statistically significant and positive effect of this factor on processing time. Moreover, the digital cumulative team has a factor load of 1 relative to 0.7 for the physical cumulative team. This factor is statistically insignificant for communication time. Thus, as far as cumulative scale is concerned, the digital team has the larger effect on hospitalist processing time. This confirms and quantifies intuition: the larger the digital team the more information is accumulated in the patient EHR and the more data the hospitalist must read and process. This is a macro effect.

\section{Workflow interruptions}

In contrast to team scale, workflow interruptions are purely a physical team effect. They are captured by our second factor $\mathrm{G}_{2}$ (workflow interruptions) which features no digital-layer variables. When the hospitalist's consults a cardiologist, the latter may

${ }^{4}$ The EHR data covers a wider date range than the observed data so that some patients' first observed days are not their admission days.

${ }^{5}$ Our model predicts an average processing time of $26.7 \mathrm{~min}$ given the actual data, which is close to the observed average of $26.1 \mathrm{~min}$. Note that the prediction of the average log (processing time) equals the observed average log (processing time). 
not be immediately available and only later contacts back the hospitalist, thereby possibly interrupting the hospitalist's workflow. The regression shows that this factor is statistically significant and increases both processing time and communication time. There are two mechanisms that could explain this effect: First, the larger the physical team the more time spent on communication and the more information that the hospitalist must process. The second mechanism, investigated rigorously in [29], is the increase in processing time due to the mental setup time introduced by interruptions.

\section{Team instability factor}

$G_{3}$ increases processing time in a statistically significant way but is statistically insignificant for communication time. The effects of team instability can be decomposed into team unfamiliarity and task unfamiliarity. Intuitively, less familiarity among team members captured by a higher physical new-member fraction and a lower collaboration experience-may render the exchange of information less efficient and require the hospitalist to spend more time processing the input. Our factor analysis suggests, however, that task/patient unfamiliarity, and not member unfamiliarly, is the more prominent effect of instability. The physical new-member fraction and the digital daily team size (which contains $70 \%$ new team members) has a combined factor loading of $0.7+0.4=1.1$ while collaboration experience (a proxy for member unfamiliarity) has a factor loading of 0.4 . I.

\section{Limitations}

While our team evolution model is general, the empirical application is specific to hospitalist workflows and only serves as a "proof of concept;" i.e., an example of the type of insights it can provide. Some caveats are in order. First, the observational study captures 17 days and 4 hospitalists working at $\mathrm{NMH}$. While we were assured that there was no bias in the selection of observation days and observed hospitalists and while a power analysis shows that 50 observed patient-day observations would suffice (we have 229), additional observation days and hospitalists would, of course, improve fidelity but also increase researcher cost. We believe we struck the right balance for our purposes as our standard errors are sufficiently small. While our estimated numeric values provide evidence of the order of magnitude of team effects, they are specific to NMH and may change at other locations. Second, we recognize that hospitalist processing times address only one part of the total effectiveness of quality patient care. We have not addressed how hospitalist processing time could be improved to approach the best possible benchmark without negatively impacting other caregivers or the total quality of patient care. The goal of this paper was to provide a data-driven methodology to evaluate and estimate the best possible impact of team interaction on an individual caregiver's processing time. Future work should study the total team effective productivity in other health care settings.

\section{Conclusion}

This paper demonstrates that team interaction determines almost three quarters of the total daily time a hospitalist spends on a patient while patient characteristics affect only one quarter. In addition, despite the absence of interpersonal communication with the focal team member, digital team members have almost equal impact on hospitalist processing time as physical team members. Analysis thus requires merging observational small data (time-motion study) with digital big data (EHR).

Team interaction is multi-faceted and encompasses many variables. Our methodology groups the many team variables into a small number of team factors that predict individual processing time. These factors are naturally interpreted as team size, team stability, and workflow interruptions. This approach and associated benchmarks for team interaction should be useful in any environment where team interaction is essential.

\section{Supplementary Information}

\section{References}

1. Benhiba L, Loutfi A, Janati Idrissi MA (2017) A Classification of Healthcare Social Network Analysis Applications In: Proceedings of the $10^{\text {th }}$ International Joint Conference on Biomedical Engineering Systems and Technologies, 10.5220/0006168001470158.

2. Carson MB, Scholtens DM, Frailey CN, Gravenor SJ, Kricke GE, et al. (2016) An outcome-weighted network model for characterizing collaboration PLoS One 11: $\mathrm{e} 0163861$.

3. Soulakis ND, Carson MB, Lee YJ, Schneider DH, Skeehan CT, et al. (2015) Visualizing collaborative electronic health record usage for hospitalized patients with heart failure. J Am Med Inform Assoc 22: 299-311.

4. Kilner E, Sheppard LA (2010) The role of teamwork and communication in the emergency department:a systematic review. Int Emerg Nurs 18: 127-37.

5. Uddin S, Hossain L, Hamra J, Alam A (2013) A study of physician collaborations through social network and exponential random graph. BMC Health Serv Res 13: 234 .

6. Wachter RM, Goldman L (1996) The emerging role of "hospitalists" in the american health care system. N Engl J Med 335: 514-7.

7. Elliott DJ, Young RS, Brice J, Aguiar R, Kolm P (2014) Effect of hospitalist workload on the quality and efficiencyof care. JAMA Intern Med 174: 786-93.

8. Contractor NS, Monge PR (2002) Managing knowledge networks. Manag Commun Q 16: 249-58.

9. Kim T, McFee E, Olguin DO, Waber B, Pentland A, et al. (2012) Socio- metric badges: Using sensor technology to capture new forms of collaboration. J Organ Behav 33: 412-27.

10. Edmondson AC, Winslow AB, Bohmer RMJ, Pisano GP (2003) Learning how and learning what: Effects of tacit and codified knowledge on performance improvement following technology adoption. Decis Sci 34: 197-224.

11. Guimerà R, Uzzi B, Spiro J, Amaral LA (2005) Team assembly mechanisms determine collaborationnetwork structure and team performance. Science 308: 697-702. 
12. Team membership categories establish the team evolution model, which is explained in materials and methods as supplementary materials.

13. Newman MEJ (2001) Clustering and preferential attachment in growing networks. Phys Rev E 64: 025102.

14. Barabasi AL, Jeong H, Neda Z, Ravasz E, Schubert A, et al. (2002) Evolution of the social network of scientific collaborations. PHYSICA A 311 : 590-614.

15. Holme P, Edling CR, Liljeros F (2004) Structure and time evolution of an internet dating community. Soc Networks 26: 155-74.

16. Kossinets G, Watts DJ (2006) Empirical analysis of an evolving social network. Science 311: 88-90.

17. Christakis NA, Fowler JH (2007) The spread of obesity in a large social network over 32 years. N Engl J Med 357: $370-9$.

18. Christakis NA, Fowler JH (2008) The collective dynamics of smoking in a large social network. N Engl J Med 358: $2249-58$.

19. Palla G, Barabási AL, Vicsek T (2007) Quantifying social group evolution. Nature 446: 664-7.

20. Rand DG, Arbesman S, Christakis NA (2011) Dynamic social networks promote cooperation in experiments with humans. Proc Nat Acad Sci USA 108: 19193-8.

21. Sample data collected from the time-motion study is displayed in supplementary materials.

22. Data collection and merging are explained in methods and materials as supplementary materials.

23. Variable definitions and measurements are available in supplementary materials.

24. Acuity, assessed by the admitting physician, ranges from 1 to 5 , with 5 being the most acute. $84 \%$ of patients in our data have acuity of 3 or lower. Acuity of the patient may affect the base documentation time as well the need to reach out to specialists.

25. Robustness checks on altering the constraint of number of factors are available in supplementary materials.

26. Personality Project (2015) William Revelle \& Maintainer William Revelle, Package apsycha, USA.

27. Propensity score weighting and GLM are explained in supplementary materials.

28. Robustness checks on regression models are displayed in supplementary materials.

29. Gurvich I, O’Leary KJ, Wang L, Van Mieghem JA (2017) Collaboration, inter- ruptions and changeover times: Model and empirical study of hospitalist processing times. SSRN: 10.2139/ssrn.2616926.

30. McCaffrey DF, Ridgeway G, Morral AR (2004) Propensity score estimation with boosted regression for evaluating causal effects in observational studies. Psychol Methods 9: 403-25.

31. Decomposition procedures are explained in supplementary materials.

32. Decomposition procedures are explained in supplementary materials.

33. Predictions of processing times under the four team scenarios are explained in supplementary materials.

34. Factor analysis and more robustness checks are available in supplementary materials.

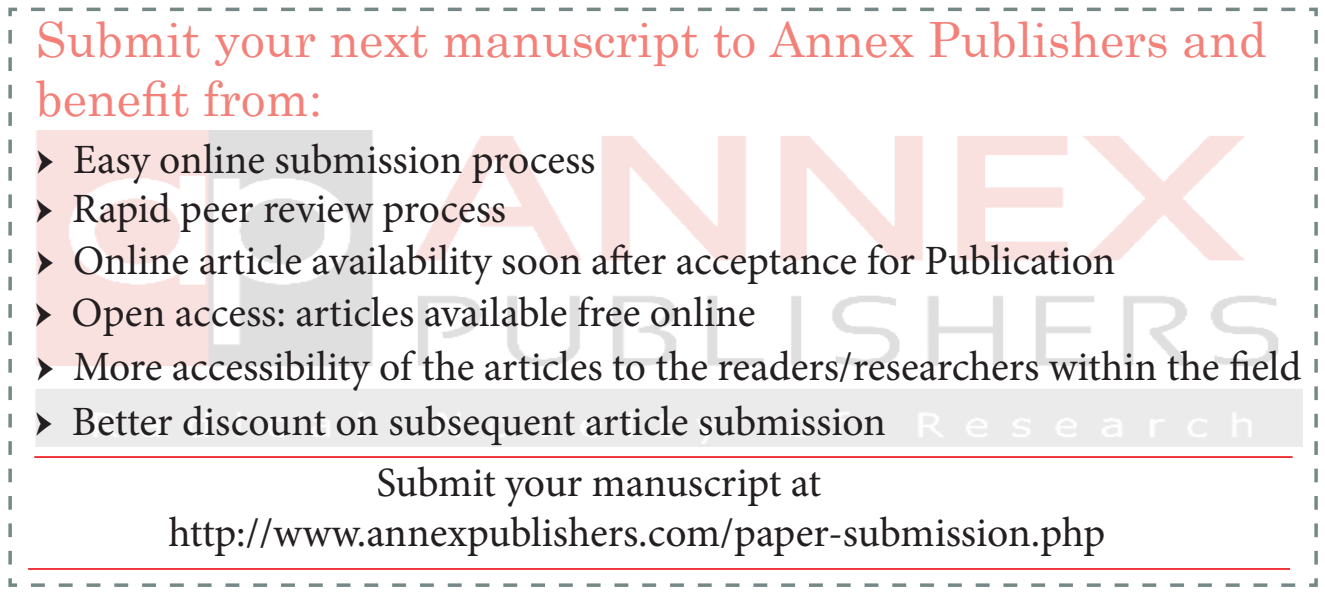

